\title{
NUEVA GUÍA TECNICA UEAtc PARA LA EVALUACIÓN DE LAS COLAS PARA REVESTIMIENTOS CERÁMICOS (COMENTARIOS).
}

\author{
(NEW UEATC TECHNICAL GUIDE FOR EVALUATING CERAMIC COATING GLUES)
}

César del Olmo Rodríguez

Dr. en Ciencias Químicas, ICCET/CSIC

Fecha de recepción: $25-X-90$ ESPAÑA

\section{RESUMEN}

En el presente artículo se analiza, con motivo de su reciente publicación, la nueva Guía Técnica UEAtc para la evaluación de las colas para revestimientos cerámicos, de Marzo de 1990, destacando las novedades recogidas en este documento técnico y las diferencias con respecto a las anteriores Directrices, de Julio de 1979, a las que reemplaza y anula. Asimismo, como información, se incluyen 2 cuadros donde se resumen las nuevas especificaciones y el control de calidad para la fabricación de estos productos que se define en el documento.
SUMMARY:

This article analyzes the new UEAtc Technical Guide for evaluating ceramic coating glues, on the occasion of its publication, in March 1990, pointing out the novelties included in this technical document and the differences compared with the previous Directives of July 1979, which they replace and annul.

Likewise, two informative charts are included, which summarize the new specifications and quality control for manufacturing these products, as defined in the document.

\section{INTRODUCCIÓN}

En el mes de marzo de 1990 ha sido aprobado en el seno de la Unión Europea para la Idoneidad Técnica en la Construcción (UEAtc), organismo técnico al que España pertenece como miembro fundador*, la nueva Guia Ténica UEAtc, que contiene las especificaciones para evaluar las colas para revestimientos cerámicos, y que sirve de guía para la concesión del Documento de Idoneidad Técnica (D.IT.) a estos materiales de construcción no tradicionales. Este documento anula y reemplaza a las antiguas Directrices UEAtc vigentes desde el año 1979.

\section{CONTENIDO DEL NUEVO DOCUMENTO TÉCNICO}

La Guia Técnica UEAtc que se comenta, está dividida en los siete capítulos siguientes: 1 . Introducción, 2 . Reglas generales de calidad, 3. Terminología, 4. Métodos

\footnotetext{
* El Instituto de Ciencias de la Construcción Eduardo Torroja es el representante de España en este Organismo.
}

de ensayo, 5. Especificaciones, 6. Control de calidad y 7. Contenido del Documento de Idoneidad Técnica.

Las colas se clasifican en el documento según su uso, definiéndose por separado las que se destinan a la colocación de revestimientos cerámicos sobre los cuatro elementos constructivos siguientes:

- muros interiores

- suelos interiores

- muros exteriores, y

- suelos exteriores,

estableciéndose para cada uno de ellos:

- las reglas generales de calidad

- las especificaciones, y

- el control de calidad

Respecto a los métodos de ensayo, la mayoría de éstos se definen con carácter general para las colas destinadas a todos los usos mencionados; junto a estos métodos experimentales, se incluyen también proce- 
dimientos operatorios que son específicos para un uso determinado. Con carácter específico, la nueva Guía Técnica contiene un número de ensayos muy superior a la precedente.

\section{PRINCIPALES NOVEDADES}

La Guia Técnica UEAtc que se comenta, contiene importantes novedades con respecto a la anterior, que se analizan por capitulos.

\subsection{Materiales}

La nueva Guia Técnica contempla mayor número de materiales (colas) que las consideradas en el documento precedente, al haberse recogido en ésta una serie de productos que han aparecido en el mercado europeo en los últimos años.

El cuadro I recoge, de forma resumida, estas diferencias apuntadas.

\section{Cuadro I}

\begin{tabular}{|c|c|}
\hline $\begin{array}{l}\text { Directrices UEAtc } \\
\text { (año 1979) }\end{array}$ & $\begin{array}{l}\text { Guía Técnica UEAtc } \\
\text { (año 1990) }\end{array}$ \\
\hline \multicolumn{2}{|c|}{$\begin{array}{l}\text { Clasificación de las colas para } \\
\text { revestimientos cerámicos }\end{array}$} \\
\hline $\begin{array}{l}\text { - Morteros cola } \\
\text { - Cementos cola } \\
\text { - Cementos cola de } \\
2 \text { componentes } \\
\text { - Adhesivos sin } \\
\text { cemento listos } \\
\text { para el uso } \\
\text { - Otros adhesivos* }\end{array}$ & $\begin{array}{l}\text { - Colas de endureci- } \\
\text { miento hidráulico } \\
\text { - Colas de endureci- } \\
\text { miento hidráulico } \\
\text { con adición de un } \\
\text { ligante (orgánico) } \\
\text { - Colas especiales } \\
\text { de endurecimiento } \\
\text { hidráulico } \\
\text { para suelos } \\
\text { - Colas en solución } \\
\text { (dispersión acuosa) } \\
\text { - Cementos cola } \\
\text { con caseina } \\
\text { - Sistemas de } 2 \\
\text { componentes en } \\
\text { fase reactivo* }\end{array}$ \\
\hline
\end{tabular}

* No tratados en el documento, aunque sí se mencionan en él.

\subsection{Ensayos}

Otra novedad importante que contiene este documento se incluye en el capítulo de ensayos.
Se definen, por primera vez, los ensayos relativos a:

- Comportamiento de la película de cola al ensayo de flexión (específico para las colas de endurecimien. to hidráulico con adición de un ligante orgánico).

- Apreciación de la retracción (para adhesivos en capa gruesa).

- Apreciación de las tolerancias en el agua de amasado (para los productos preparados para su mezcla con agua).

- Penetración de la aguja (para los cementos cola con caseina*).

- Apreciación de la consistencia de la cola, que representa otra forma de evaluar el aplastamiento de los surcos de cola**.

Asimismo, algunos de los ensayos incluidos en las $\mathrm{Di}$ rectrices anteriores, se realizan en esta Guía Técnica según métodos de ensayo algo diferentes, lo que influye en los resultados que se obtienen.

Los nuevos métodos de ensayo se refieren a la evaluación de:

\section{- El tiempo abierto teórico}

Dos novedades importantes se han introducido en el ensayo. La primera, se refiere a la forma de colocación de las plaquetas que, en el nuevo método, y tras el mismo procedimiento inicial seguido en el método anterior (extendido de la cola sobre el soporte, peinado de ésta con la espátula dentada para regular el espesor, y aplicación sobre las plaquetas de un peso de $2 \mathrm{~kg}$ durante $15 \mathrm{seg}$ en este caso), se someten éstas inmediatamente después, en primer lugar, a un giro, que rompe, parcial o totalmente, la película de cola que pudiera haberse formado sobre la superficie de la misma (por cuya operación, en general, se produce un aumento de la adherencia), $y$, a continuación, las plaquetas se cargan de nuevo con un peso de $2 \mathrm{~kg}$ que se mantiene durante $15 \mathrm{seg}$.

\footnotetext{
* Este ensayo constituye una forma de medir la velocidad de fraguado de estas colas, cuyo endurecimiento se produce a través de un proceso de secado.

* * Este ensayo determina el valor de la masa, en Newton - N-, necesaria para producir (por efecto del aplastamiento de dicha masa) una película contínua en el mortero cola, aún fresco, aplicado sobre el soporte; operación que se realiza al cabo de un tiempo equivalente al tiempo abierto teórico (de 20 a 30 minutos, según el tipo de cola). En las especificaciones se fija, para cada familia de colas, el valor máximo que deberá tener esta masa.
} 
La segunda novedad se refiere a los tiempos establecidos para realizar el ensayo, que, en este caso, son: a) el tiempo abierto anunciado por el fabricante y b) 10 minutos después del tiempo anterior.

- La adherencia inicial (sin tratamientos), que incluye, para determinados tipos de cola, un ensayo a las edades de:

- 1 día y

- 3 dias

- La adherencia después de la acción del agua, que establece dos procedimientos de ensayo diferentes, según sea la resistencia a la humedad de la cola evaluada.

Por último, los ensayos restantes permanecen sin modificaciones.

\subsection{Especificaciones y control de calidad}

Las diferencias entre ambos documentos, con respecto a estos dos capítulos, se basan principalmente en que, uno y otro, se refieren a materiales algo diferentes. Se prescinde de profundizar en estas cuestiones, al quedar éstas explicitadas en los Cuadros II y III que se incluyen, como información, en los dos apartados 4 y 5 siguientes.

\subsection{Contenido del Documento de Idoneidad Técnica}

Como novedad, con respecto al documento anterior, la vigente Guía Técnica UEAtc concreta la relación de datos e información mínima que debe incluir el D.I.T. de las colas para revestimientos cerámicos.

\section{ESPECIFICACIONES}

En el cuadro II se resumen las especificaciones contenidas en la nueva Guia Técnica UEAtc para la evaluación de las colas para revestimientos cerámicos.

El cuadro II se divide en 4 bloques o grandes columnas. La primera columna, se refiere a los 5 tipos de colas definidos en el documento, anteriormente mencionadas.

El segundo bloque, agrupa las especificaciones que guardan relación con las características de TRABAJABILIDAD de la cola. Se incluyen entre éstas: la apreciación del Tiempo abierto, la medida del deslizamiento sobre pared vertical (que se establece con carácter es- pecífico para el uso de la cola en estas condiciones - paramentos verticales - el tiempo de vida en el bote (potlife) y la consistencia de la pasta. Asimismo, para las colas a mezclar con agua, se fijan (mediante ensayos oportunos) las tolerancias admitidas en el agua de amasado, sobre la proporción indicada por el fabricante.

El tiempo abierto deberá ser, en general, al menos, igual al anunciado por el fabricante y, como mínimo, de 20 min para las colas de endurecimiento hidráulico, normales y especiales para suelos, y de 30 min para las colas restantes.

El tiempo de vida de la cola en el bote está especificado únicamente para los morteros cola especiales de fraguado rápido, para suelos, estableciéndose que la duración útil de la cola deberá ser, al menos, la anunciada por el fabricante.

El tercer bloque corresponde a los ensayos que, en general, se denominan de APTITUD PRELIMINAR que llevan el nombre de Ensayos de Identificación, en el caso de las colas especiales de endurecimiento hidráulico para suelos.

El tercer bloque comprende los ensayos siguientes: Pérdida de agua*, comportamiento a flexión de la pelicula de cola, especificado únicamente para las colas de endurecimiento hidráulico con adición de un ligante (orgánico); medida de la retracción , con carácter general, excepto para los cementos cola con caseina, aunque tampoco rige para los morteros cola corrientes; $y$, por último, la penetración de la aguja (de Vicat), para los cementos cola con caseina únicamente.

El cuarto y último bloque, se refiere a los ensayos que, en general, se denominan de APTITUD DE EMPLEO; aunque, en el caso de las colas especiales de endurecimiento hidráulico para suelos, reciben el nombre de Ensayos de conservación de la calidad.

Este bloque comprende los ensayos de adherencia, de resistencia al cizallamiento y de comportamiento al choque.

Los ensayos de adherencia se realizan, en primer lugar, sin tratamiento, después de varios períodos de conservación (3 días y 28 días) en general para todas las colas; pero, se excluye, a 3 dias, para los morteros cola corrientes, para los adhesivos sin cemento corrientes, y también para los cementos cola con caseina; sin embargo, y por el contrario, los morteros cola de fraguado rápido se ensayan además a 1 dia.

* Medida a través del ensayo de "poder de retención de agua". http://informesdelaconstruccion.revistas.csic.es 
Cuadro II

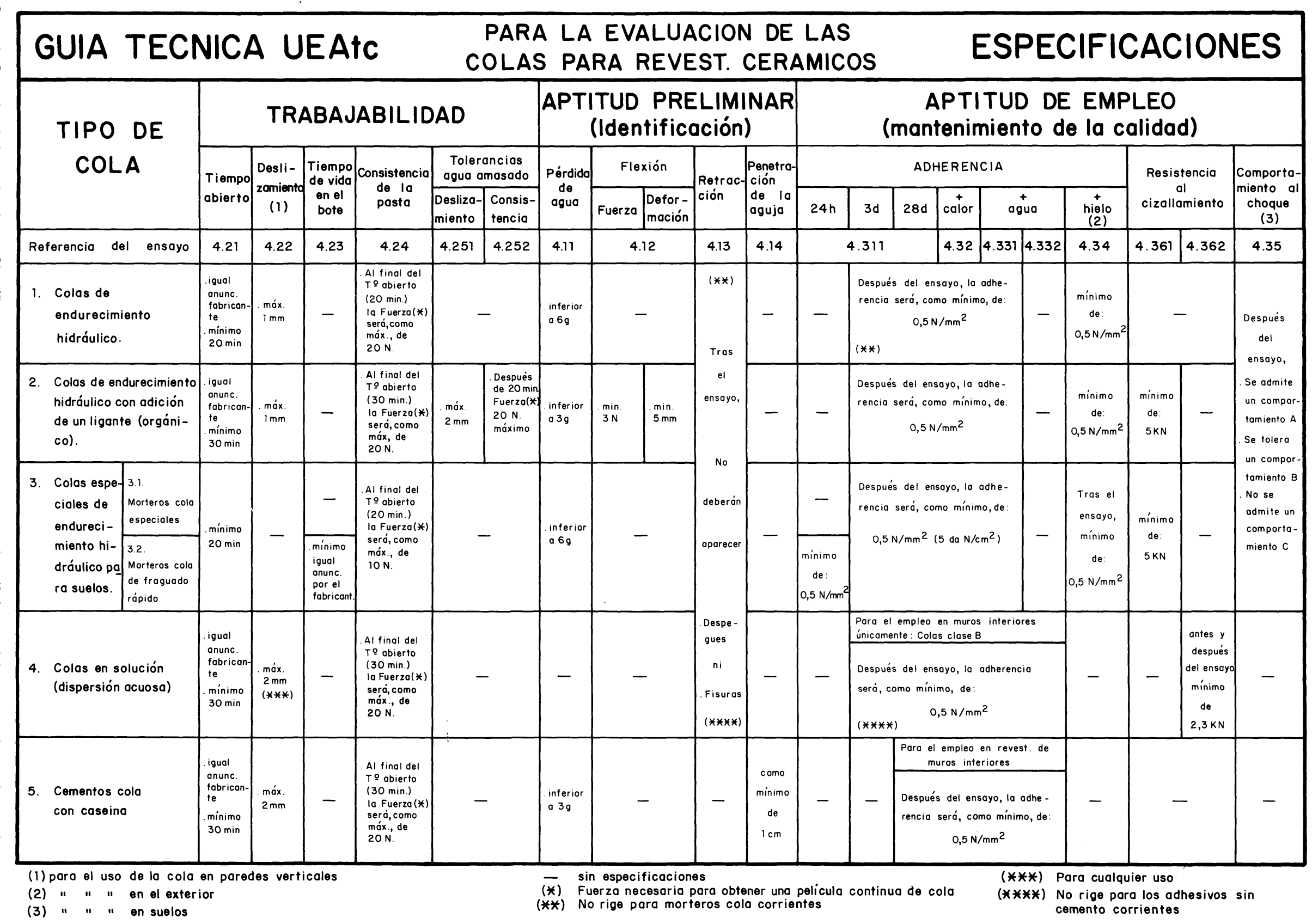


Cuadro III

\begin{tabular}{|c|c|c|c|c|c|}
\hline GUIA TECN & UEA & $\begin{array}{r}\text { PARA L } \\
\text { COLAS P }\end{array}$ & $\begin{array}{l}\text { EVALUACION } \\
\text { ARA REVEST. CE }\end{array}$ & $\begin{array}{l}\text { LAS } \\
\text { AMICOS }\end{array}$ & c \\
\hline \multirow{4}{*}{\multicolumn{2}{|c|}{$\begin{array}{l}\text { TIPO DE } \\
\text { COLA }\end{array}$}} & \multicolumn{3}{|c|}{ CONTROL INTERNO DEL FABRICANTE } & \multirow{3}{*}{$\begin{array}{l}\text { SUPERVISION EVENTUAL DEL } \\
\text { AUTOCONTROL POR UN } \\
\text { ORGANISMO INDEPENDIENTE }\end{array}$} \\
\hline & & \multirow{2}{*}{$\begin{array}{l}\text { RECEPCION DE } \\
\text { MATERIAS PRIMAS } Y \\
\text { COMPONENTES: } \\
\end{array}$} & \multicolumn{2}{|c|}{ PRODUCTO TERMINADO } & \\
\hline & & & CONTROL EXPEDICION & CONTROL INFORMACION & \\
\hline & & MATERIALES A CONTROLAR: & \multicolumn{2}{|c|}{ ENSAYOS A REALIZAR } & ENSAYOS A REALIZAR \\
\hline \multicolumn{2}{|c|}{$\begin{array}{l}\text { 1. COLAS DE ENDURECIMIENTO } \\
\text { HIDRAULICO }\end{array}$} & $\begin{array}{l}\text { Polvo (filler) } \\
\text { Cemento } \\
\text { Arena } \\
\text { Aditivos }\end{array}$ & \multirow{4}{*}{$\begin{array}{l}\text { Ensayo simplificado de } \\
\text { adherencia } \\
\text { Consistencia de la cola } \\
\text { y transferencia }\end{array}$} & \multirow{6}{*}{$\begin{array}{l}\text { Frecuentemente } \\
\text { Tiempo abierto } \\
\text { Deslizamiento } \\
\text { Adherencia } \\
\text { Con frecuencia menor } \\
\text { Los demás ensayos } \\
\text { de aptitud de empleo } \\
\text { y trabajabilidad. }\end{array}$} & $\begin{array}{l}\text { Retención de agua } \\
\text { Tiempo abierto } \\
\text { Adherencia }\end{array}$ \\
\hline \multirow{2}{*}{$\begin{array}{l}\text { 2. COLAS ESPECIALES } \\
\text { DE ENDURECIMIENTO } \\
\text { HIDRAULICO CON ADI- } \\
\text { CION DE UN LIGANTE } \\
\text { (ORGANICO) }\end{array}$} & $\begin{array}{l}\text { MORTERO COLA } \\
\text { CON LIGANTE MIXTO }\end{array}$ & $\begin{array}{l}\text { Polvo (filler) } \\
\text { Cemento } \\
\text { Arena } \\
\text { Aditivos }\end{array}$ & & & $\begin{array}{l}\text { Retención de agua } \\
\text { Tiempo abierto } \\
\text { Adherencia } \\
\text { Resistencia al cizallamiento }\end{array}$ \\
\hline & $\begin{array}{l}\text { MORTERO COLA } \\
\text { CON RESINA } \\
\text { LIQUIDA }\end{array}$ & $\begin{array}{l}\text { Polvo (filler) } \\
\text { Cemento } \\
\text { Arena } \\
\text { Aditivos } \\
\text { Resina } \\
\text {. Extracto seco } \\
\text {. Viscosidod } \\
\text {. pH }\end{array}$ & & & $\begin{array}{l}\text { Retención de agua } \\
\text { Tiempo abierto } \\
\text { Adherencia }\end{array}$ \\
\hline \multicolumn{2}{|c|}{$\begin{array}{l}\text { 3. COLAS ESPECIALES DE ENDURECIMIENTO } \\
\text { HIDRAULICO PARA SUELOS }\end{array}$} & $\begin{array}{l}\text { Polvo (filler) } \\
\text { Cemento } \\
\text { Arena } \\
\text { Aditivos }\end{array}$ & & & $\begin{array}{l}\text { Retención de aguo } \\
\text { Tiempo abierto } \\
\text { Adherencio }\end{array}$ \\
\hline \multicolumn{2}{|c|}{$\begin{array}{l}\text { 4. COLAS EN SOLUCION } \\
\text { (EN DISPERSION ACUOSA) }\end{array}$} & $\begin{array}{l}\text { Resina } \\
\text {. Extracto seco } \\
\text { Viscosidad } \\
\text {. pH } \\
\text { Cargas } \\
\text { Granulometría }\end{array}$ & $\begin{array}{l}\text { Ensayo simplificado de } \\
\text { adherencio }\end{array}$ & & $\begin{array}{l}\text { Tiempo abierto } \\
\text { Adherencia } \\
\text { Retracción (para los productos que } \\
\text { se aplican en capa gruesa) }\end{array}$ \\
\hline \multicolumn{2}{|c|}{ 5. CEMENTOS COLA CON CASEINA } & $\begin{array}{l}\text { Polvo (filler) } \\
\text { Cemento } \\
\text { Arena } \\
\text { Aditivos }\end{array}$ & $\begin{array}{l}\text { Ensayo simplificado de } \\
\text { adherencia } \\
\text { Consistencia de la cola } \\
\text { y transferencia }\end{array}$ & & $\begin{array}{l}\text { Retención de agua } \\
\text { Tiempo abierto } \\
\text { Adherencio } \\
\text { Penetración de la aguja }\end{array}$ \\
\hline
\end{tabular}

Asimismo, la adherencia se mide también después de diversos tratamientos: después de la acción del calor, después de la acción del agua y después de ciclos de hielo-deshielo, en este último caso, para el uso de la cola en el exterior.

Después de los ensayos anteriores, la adherencia deberá ser, en todos los casos, como mínimo de 0,5 $\mathrm{N} / \mathrm{mm}^{2}$ (5 daN/ $\left./ \mathrm{cm}^{2}\right)$.

La resistencia al cizallamiento, se especifica en 3 de los tipos de colas incluidos en el documento, excluyéndose para las colas de endurecimiento hidráulico y para los cementos cola con caseina.

Por último, el comportamiento al choque se evalúa para el uso de la cola en suelos, estableciéndose que, para las 3 primeras colas incluidas en el documento, se admite un comportamiento tipo A, se tolera un compor- tamiento tipo $B$ y no se admite un comportamiento tipo $C^{*}$

\section{CONTROL DE CALIDAD}

En el cuadro III se extractan los ensayos que comprende el control interno del fabricante (autocontrol), asi como las comprobaciones que deben efectuarse para la supervisión eventual del autocontrol por un organismo

\footnotetext{
* Tras la caida de la bola en el ensayo, se pueden observar los com. portamientos siguientes:

Comportamiento $A$ : únicamente resulta visible a simple vista el impacto circular y no se observan fisuras sobre la plaqueta.

* Comportamiento B: el impacto circular aparece rodeado de fisuras dispuestas en estrella.

* Comportamiento C: la losa, completamente rota, se despega por trozos y a veces estalla

http://informesdelaconstruccion.revistas.csic.es
} 
independiente, definidos en el capítulo 6 del documento, que corresponden a la fabricación de una cola para acogerse al Documento de Idoneidad Técnica (D.IT.).

El cuadro III se divide en 3 bloques o grandes columnas. La primeras columna, se refiere a los 5 tipos de colas definidos en la Guía Técnica UEAtc.

El segundo bloque, agrupa los ensayos de recepción de las materias primas y componentes y los ensayos que deben realizarse sobre el producto terminado, dividiéndose éstos en ensayos de control de expedición y de control de la información*.

La recepción de las materias primas y componentes debe abarcar a todos los constituyentes que entran a formar parte de la cola (cemento, arena, filler, cargas y aditivos); no se concreta, sin embargo, los ensayos que deberán realizarse con esta finalidad en el caso de los materiales que se comercializan en polvo, aunque si se especifica para la resina, en el caso de los morteros cola con resina líquida y de las colas en solución.

El control de expedición deberá incluir un ensayo simplificado de adherencia y la apreciación de la consistencia de la cola y de la transferencia.

Por su parte, el control de la información comprende: la realización, con cierta frecuencia, de la apreciación del Tiempo abierto, deslizamiento y adherencia, y, con

\footnotetext{
* Este control tiene por objeto comprobar que los controles funcio nan normalmante y no se produce ningún hecho anormal en la fabricación; y que la calidad del producto coincide con la exigida en la Guía Técnica.
}

una frecuencia menor, de los demás ensayos de Aptitud de empleo y de Trabajabilidad.

Por último, el tercer bloque, contiene la relación de ensayos de comprobación que incluye la supervisión eventual del autocontrol por un organismo independiente.

Estos ensayos son, con carácter general, retención de agua, tiempo abierto y adherencia.

Además de estos ensayos, se deberán incluir también, la medida de la resistencia al cizallamiento, para las colas especiales de endurecimiento hidráulico con adición de un ligante orgánico y la penetración de la aguja (de Vicat) para los cementos cola con caseina.

Por último, en el caso de las colas en solución, se suprime el ensayo de retención de agua y se incluye el ensayo de retracción.

\section{BIBLIOGRAFIA}

Directrices UEAtc para la evaluación de las colas para revestimientos cerámicos. Año 1979. Informes de la Construcción n. 349 y 350. Mayo y Junio de 1983.

Guía Técnica UEAtc para la evaluación de las colas para revestimientos cerámicos. Año 1990. Monografía n. 403 del Instituto de Ciencias de la Construcción EDUARDO TORROJA.

\title{
publicación del ICCET/CSIC
}

\author{
ALOJAMIENTO Y TECNOLOGÍA: \\ ¿INDUSTRIALIZACIÓN ABIERTA? \\ Julián Salas Serrano
} La experiencia nacional en construcción industrializada en la última década, aunque no abundante,
puede resultar paralizante. Como reacción, este trabajo trata de elaborar y ordenar la información que, pegado al terreno, se ha acumulado durante los sesenta.

Auscultando tendencias avanzadas en otros paises y apoyándose en nuestra realidad cotidiana, el autor de este trabajo apuesta por la industrialización, presintiendo un futuro con pocos puntos en común con lo que en general, hasta bien reciente, se ha conocido como construcción industrializada.

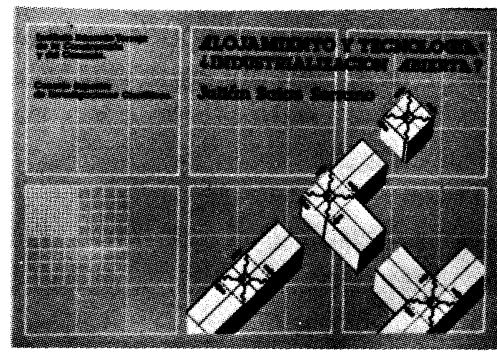

Se abre el trabajo con un prólogo del Profesor Ciribini en el cual, con rigor conceptual y desde su rica experiencia, analiza algunos de los conceptos más polémicos del fenómeno de industrialización del sector construcción. El autor recopila un conjunto de aportaciones de maestros de la arquitectura al lento proceso de evolución tecnológica y conceptual, continuando con un intento de respuesta realista a la interrogante que flota en el sector nacional: ¿réquiem por la industrialización?

Se dedican otros capítulos a revisar lo realizado y a encarar el futuro inmediato: el concepto de componente, su repercusión económica, la dificultad del proyecto a base de productos industriales, la nueva vigencia embrionaria de la construcción por medio de catálogos, los conceptos de flexibilidad e intercambiabilidad desde una óptica práctica... Especial atención dedica este trabajo a la exposición de lo que podiamos llamar nuevos derroteros de la coordinación dimensional, aportando una visión actualizada y pragmática de los enfoques más implantados en Europa.

Las siempre problemáticas interrelaciones entre normativa, calidad e industria se detallan de forma documentada y realista. Finaliza el trabajo con unas reflexiones dirigidas al ámbito empresarial y un esbozo de "reglas de juego" que faciliten al subsector la salida del uimpasse» actual.

Un volumen encuadernado en rústica, de $24 \times 16,5 \mathrm{~cm}$, compuesto de 160 páginas, con 109 figuras, 19 tablas y 86 referencias bibliográficas. Madrid, junio, 1981 . 